\title{
Causative organisms and outcomes of peritoneal dialysis-related peritonitis in Sarawak General Hospital, Kuching, Malaysia: a 3-year analysis
}

Vui Eng Phui ${ }^{1 *}$, Clare Hui Hong Tan ${ }^{1}$, Chee Kean Chen ${ }^{2}$, Kee Hoe Lai ${ }^{3}$, Kwek Foong Chew ${ }^{1}$, Hock Hin Chua ${ }^{1}$, Laura Lui Sian $\mathrm{Ngu}^{1}$ and Lawrence Wei Soon $\mathrm{Hii}^{1}$

\begin{abstract}
Background: Peritoneal dialysis peritonitis remains a significant cause of morbidity for peritoneal dialysis patients and the main reason for conversion from peritoneal dialysis to hemodialysis. As the characteristics of patients and microbial susceptibility vary from center to center, the aim of this study is to evaluate the microbiology and the clinical outcomes among continuous ambulatory peritoneal dialysis patients in Kuching, Malaysia.

Methods: This is a retrospective record review of 82 continuous ambulatory peritoneal dialysis patients who developed peritonitis during 2013 to 2015. Data examined included patients' demographic data, causative organisms, and outcomes.
\end{abstract}

Results: A total of 124 episodes of peritonitis were recorded, and the overall peritonitis rate was 0.40 episodes per patient-year. There was an increasing incidence in continuous ambulatory peritoneal dialysis peritonitis over the 3-year study period ( 0.35 to 0.47 episodes per patient-year). The gram-negative peritonitis rate increased over the period until towards the end of the study period, when gram-positive and gram-negative organisms accounted for almost equal proportions of peritonitis. Streptococcus sp. was the most common organism among the gram-positive peritonitis while Pseudomonas sp. was the most common organism in gram-negative peritonitis. The culture-negative peritonitis rate was $25.8 \%$. The peritoneal dialysis catheter was removed in 32 episodes (26.6\%). The catheter loss rate was significantly higher in gram-negative peritonitis, as compared to gram-positive peritonitis (38.9 vs 16.7\%, $p=0.027$ ).

Conclusions: The increasing trend of peritonitis and high rates of culture negativity and peritoneal dialysis catheter removal are areas that need further evaluation and improvement in the future. Study on risk factors of continuous ambulatory peritoneal dialysis peritonitis, detailed microbiology, and antimicrobial treatment and response are warranted to further improve the outcomes of continuous ambulatory peritoneal dialysis patients.

Keywords: Continuous ambulating peritoneal dialysis, Peritonitis, Microorganisms, Catheter removal

\footnotetext{
* Correspondence: leshally@hotmail.com

${ }^{1}$ Department of Medicine, Sarawak General Hospital, Jalan Hospital, 93586

Kuching, Sarawak, Malaysia

Full list of author information is available at the end of the article
} 


\section{Background}

Peritoneal dialysis (PD) is an important treatment modality for patients with end-stage renal disease (ESRD) [1-4]. Despite the introduction of improved connection systems and PD solutions, peritonitis remains a significant cause of morbidity for PD patients and the main reason for conversion from PD to hemodialysis [4-7].

The clinical outcome of PD peritonitis is determined greatly by the causative organism $[8,9]$. Empirical treatment usually covers both gram-positive and gramnegative organisms. However, the causative organism may vary depending on the area and the environment and social background of patients. Treatment guidelines should be modified from time to time based on knowledge of recent, local epidemiological trends and susceptibility to antimicrobial agents, to try to improve patient outcomes [10].

Sarawak is a state in Malaysia with a big geographical area and widely varied population characteristics. There is currently no published data on the causative organisms in continuous ambulatory peritoneal dialysis (CAPD) peritonitis and their outcomes in Sarawak. Hence, we studied the incidence, the microbiological characteristics, and the eventual clinical outcomes of CAPD peritonitis for all CAPD patients followed up at Sarawak General Hospital, between January 2013 and December 2015.

\section{Methods}

This is a retrospective record review of all CAPD patients on follow-up at the Nephrology Unit, Sarawak General Hospital, Kuching, Malaysia, who developed peritonitis between January 2013 and December 2015. A total of 82 CAPD patients developed peritonitis during this study period, and 124 episodes of peritonitis were recorded.

For all CAPD patients in our center, a double-cuffed straight Tenckhoff catheter was inserted by surgical technique by one of the three urologists. CAPD was started after a break-in period of $12 \pm 4$ days. All patients had routine screening for Staphylococcus aureus nasal carriage, and eradication of carriers was done by intranasal mupirocin before catheter insertion. Eight of the patients were carriers and needed the eradication. Intravenous cefoperazon and cefazolin were given as prophylactic antibiotics immediately prior to catheter insertion. All patients or their assistants underwent a structured 10 to 14 days of training at the start of CAPD. This included basic principles of CAPD, types of PD solutions and consumables, handwashing techniques and hygiene, CAPD exchange procedures and exit-site care, recording of BP, weight and ultrafiltration, peritonitis recognition and management, and diet and medication counseling. Subsequently, they were started on a disconnect system using PD solution with three 2-L exchanges daily. Forty-six patients (56.1\%) were on the Baxter system and 36 patients (43.9\%) on the Fresenius system. Either mupirocin or gentamicin cream was used on the exit site as a daily routine, and the choice was left to the discretion of the treating nephrologist.

Demographic data collected included age at time of peritonitis, sex, body mass index, education level and causes of ESRD. Clinical data pertaining to peritonitis collected included causative organisms, antimicrobial therapy, serum albumin and potassium level, intraperitoneal leucocyte count at the beginning of peritonitis, susceptibility of each organism, and date of catheter removal.

The diagnosis of PD peritonitis was made when at least two of the following three criteria were fulfilled: (i) signs and symptoms of peritonitis, (ii) cloudy dialysate with white blood cell count of $>100 / \mu \mathrm{L}$. and (iii) demonstration of organism either by smear examination or by culture of peritoneal dialysate. All 82 patients fulfilled the first and second criteria as they presented with abdominal symptoms and had cloudy dialysate with white cell count of $>100 / \mu \mathrm{L}$. An episode of peritonitis within 4 weeks after the treatment of a previous episode was considered a relapse and was not classified as a new infection. A peritonitis episode which occurred within 7 days after catheter placement was also excluded in this study. Refractory peritonitis was defined as peritonitis that did not respond to second-line antimicrobial treatment and resulted in catheter removal. The culture of PD fluid and antimicrobial sensitivity were obtained from the microbiology department of this hospital, based on hospital standard operating procedure. The causative organisms were divided into gram-positive, gramnegative, polybacterial, fungal, mycobacterium, and culture-negative.

Our first-line therapy for CAPD peritonitis was a combination of intraperitoneal cefazolin and ceftazidime. The antibiotics were adjusted later once culture and sensitivity results became available. If the peritonitis did not improve within $72 \mathrm{~h}$ and culture was negative, treatment would be escalated to IP meropenam and vancomycin. If the peritonitis still failed to respond, then the PD catheter would be removed. All patients with peritonitis also received oral nystatin or fluconazole empirically during antibiotic therapy.

Outcomes of peritonitis were classified as initial cure, catheter loss, conversion to hemodialysis, and patient death. Initial cure was defined as total resolution of peritonitis with only antimicrobial treatment. Catheter loss was defined as removal of catheter due to nonresolution of peritonitis. Patient death included any mortality within 4 weeks of presentation of peritonitis when all other possible causes of mortality had been excluded.

Peritonitis rate was calculated by totaling all the peritonitis episodes that occurred during the entire time at 
risk on CAPD for all the patients in the program during the period of the study. This total was then divided by the time at risk in years. The time at risk for peritonitis was counted from the first day of training till the occurrence of peritonitis.

Data were expressed as episodes per patient-year, percent, and mean \pm standard deviation (SD). Differences in the proportion of causative organisms were analyzed according to each calendar year. Antimicrobial susceptibilities and catheter removal rates according to the pathogens were analyzed using chi-square analysis or Fischer's exact test. Statistical significance was determined as $p$ value less than 0.05 .

\section{Results}

\section{Population characteristics}

During the study period, 124 episodes of peritonitis were detected among 82 CAPD patients. Fifty-four patients had a single episode, 18 patients had two episodes, 7 patients had three episodes, and 3 patients had four episodes of peritonitis. Gender distribution was equal with 41 male and female patients. Their mean age at the time of peritonitis was $45.4 \pm 15.8$ years. $18.3 \%$ of patients had diabetes mellitus. Twenty-eight patients (34.1\%) were on exit-site mupirocin while the remaining 54 patients (65.9\%) were on exit-site gentamicin. The baseline characteristics of patients and the underlying causes of renal impairment are shown in Table 1.

\section{Peritonitis and microbiology}

The overall peritonitis rate during the 3-year period was 0.40 episodes per patient-year. The peritonitis rate showed an increasing trend over time (Table 2). About one third (33.9\%) of the peritonitis was caused by gram-positive organisms, $29.0 \%$ by gram-negative bacteria, $3.2 \%$ were fungal infection, and $1.6 \%$ were Mycobacterium tuberculosis. Culture was sterile in $28.2 \%$ of the cases and polymicrobial in five cases (4\%).

During the 3-year period, there was an increase in peritonitis due to gram-negative organisms, from $18.8 \%$ in 2013 to $32.5 \%$ in 2014 and $32.7 \%$ in 2015 . By 2015, gram-positive and gram-negative organisms accounted for almost equal proportions of peritonitis at our center. Culture-negative peritonitis has reduced from $37.5 \%$ in 2013 to $26.9 \%$ in 2015. Peritonitis episodes secondary to fungal, $M$. tuberculosis, and polybacterial infection did not change significantly over the 3-year period.

The most common gram-positive organism responsible for CAPD peritonitis at our center was Streptococcus sp., accounting for more than one third of the gram-positive episodes, followed in descending order of frequency by coagulase-negative Staphylococcus (CoNS), S. aureus, and Enterococci. Pseudomonas aeruginosa (PAE) was the most commonly identified gram-negative organism, followed by
Table 1 Characteristics of patients

\begin{tabular}{ll}
\hline & Total \\
\hline Patients $(n)$ & 82 \\
Sex (male/female) (\%) & $41(50.0): 41(50.0)$ \\
Age (years) & $45.4( \pm 15.8)$ \\
Body mass index (kg/cm²) & $22.1( \pm 3.8)$ \\
Ethnicity (\%) & \\
Malay & $32(39.0)$ \\
Chinese & $15(18.3)$ \\
Iban & $20(24.4)$ \\
Bidayuh & $13(15.9)$ \\
Others & $2(2.4)$ \\
Living area (\%) & \\
Urban & $40(48.4)$ \\
Suburban & $42(51.6)$ \\
Education level (\%) & \\
Illiterate & $12(14.6)$ \\
Primary & $31(37.8)$ \\
Secondary & $32(39.0)$ \\
Tertiary & $7(8.6)$ \\
Underlying disease (\%) & \\
Hypertension & \\
Diabetes mellitus & $34(91.5)$ \\
Obstructive nephropathy & $15(18.3)$ \\
Gystemic lupus erythematosus & $28(34.1): 54(65.9)$ \\
Exitomerulonephritis &
\end{tabular}

Escherichia coli (E. coli), Acinetobacter spp., Klebsiella spp. (KPN), and Enterobacter.

There were four episodes (3.2\%) of fungal and two episodes $(1.6 \%)$ of $M$. tuberculosis (TB) infections during the 3-year period. Five episodes of polymicrobial peritonitis were isolated, and the combinations of infection were M. tuberculosis and CoNs, PAE and fungal infection, Acinetobacter app and Streptococcus viridans, PAE and Achromobacter spp., and KPN, E. coli, and Enterobacter. The causative organisms of peritonitis over the 3 -year study period are listed in Table 3.

Table 2 Peritonitis rate per patient-year, 2013-2015

\begin{tabular}{llll}
\hline & 2013 & 2014 & 2015 \\
\hline Cumulative patient-days & 32,177 & 39,233 & 41,289 \\
Cumulative episodes & 31 & 40 & 53 \\
Cumulative patient-months & 1057.9 & 1289.9 & 1357.4 \\
Cumulative patient case & 34.1 & 32.3 & 25.6 \\
Peritonitis episode per patient-year & 0.350 & 0.370 & 0.468 \\
\hline
\end{tabular}


Table 3 The microbiology of peritonitis episodes

\begin{tabular}{|c|c|c|c|c|c|}
\hline & $\begin{array}{l}\text { Number isolated } \\
\text { in } 2013\end{array}$ & $\begin{array}{l}\text { Number isolated } \\
\text { in } 2014\end{array}$ & $\begin{array}{l}\text { Number isolated } \\
\text { in } 2015\end{array}$ & $\begin{array}{l}\text { Total number } \\
\text { isolated } \\
(n=124)\end{array}$ & $\begin{array}{l}\text { Percentage of } \\
\text { total episode }\end{array}$ \\
\hline Gram-positive organism & $10(31.3 \%)$ & $14(35 \%)$ & $18(34.6 \%)$ & 42 & 33.9 \\
\hline Streptococcus sp. & 4 & 4 & 10 & 18 & 14.5 \\
\hline Coagulase-negative Staphylococcus (CoNS) & 4 & 5 & 5 & 14 & 13.7 \\
\hline Staphylococcus aureus & 1 & 3 & 3 & 7 & 4.0 \\
\hline Other gram-positive organisms & 1 & 2 & 0 & 3 & 1.6 \\
\hline Gram-negative organism & $6(18.8 \%)$ & $13(32.5 \%)$ & $17(32.7 \%)$ & 36 & 29.0 \\
\hline Pseudomonas sp. & 3 & 4 & 7 & 14 & 11.3 \\
\hline Escherichia coli (E. coli) & 1 & 1 & 4 & 6 & 4.0 \\
\hline Acinetobacter sp. & 1 & 2 & 2 & 5 & 4.0 \\
\hline Klebsiella sp. & 1 & 2 & 1 & 4 & 4.0 \\
\hline Enterobacter sp. & 0 & 1 & 1 & 2 & 1.6 \\
\hline Other gram-negative organisms & 0 & 3 & 2 & 5 & 6.5 \\
\hline Fungi & 2 & 1 & 1 & 4 & 3.2 \\
\hline Mycobacterium tuberculosis & 1 & 0 & 1 & 2 & 1.6 \\
\hline Polymicrobial & 1 & 3 & 1 & 5 & 4.0 \\
\hline Culture-negative & $12(37.5 \%)$ & $9(22.5 \%)$ & $14(26.9 \%)$ & 35 & 28.2 \\
\hline Total & 32 & 40 & 52 & 124 & 100.0 \\
\hline
\end{tabular}

\section{Outcome}

The PD catheter was removed in 33 (26.6\%) peritonitis episodes after failing to respond to antimicrobial treatment. Twenty-three patients were converted to hemodialysis due to the peritonitis during the study period. No death was reported in current study. Tables 4 and 5 show various causative organisms which resulted in catheter loss. The catheter loss was significantly higher in gram-negative peritonitis episodes $(p<0.05)$. Polybacterial infection also had a high rate of catheter loss (60\%). $27.8 \%$ of gram-negative peritonitis resulted in conversion to HD vs $14.3 \%$ of gram-positive peritonitis, but this did not reach statistical significance.

Among 14 episodes of Pseudomonas peritonitis, eight were on mupirocin cream, and six were on gentamicin cream. Among the six gentamicin users, four $(66.4 \%)$ gentamicin users compared with only three (37.5\%) mupirocin users needed catheter removal, as shown in Table 6.

\section{Discussion}

Peritonitis is an important cause of morbidity and mortality for CAPD patients. The epidemiology, treatment, prevention, and outcome of CAPD peritonitis have been the subject of interest among clinicians and researchers since a few decades ago [3-5]. Despite the widespread availability and awareness of both local and international guidelines for its prevention and treatment, there is still substantial variation in CAPD peritonitis microbiology and outcome among different centers and regions.

The overall CAPD peritonitis rate of 0.40 episodes per patient-year in this study is comparable to that of other centers in this region, e.g., Japan 0.195 [11], Australia 0.60 [9], Northern India 0.41 [12], Korea 0.40 [13], Thailand 0.47 [14], Singapore 0.59 [15], and Indonesia 0.25 [16]. This is however higher than the national average of 0.28 in 2014 [17]. The possible reasons could include lower educational level among our study population as $52.4 \%$ of them were either illiterate or only obtained primary education, and $51.6 \%$ were from suburban area. This may result in poorer adherence to aseptic techniques during PD exchange, suboptimal home

Table 4 Removal of PD catheter in various causative agents in peritonitis

\begin{tabular}{llll}
\hline & \multicolumn{2}{l}{ Removal of catheter } & Total \\
\cline { 2 - 3 } & Yes & No & \\
\hline Gram-positive & $7(16.7 \%)$ & $35(83.3 \%)$ & 42 \\
Gram-negative & $14(38.9 \%)$ & $22(61.1 \%)$ & 36 \\
Fungal & $3(75.0 \%)$ & $1(25.0 \%)$ & 4 \\
Tuberculosis & $2(100 \%)$ & $0(0.0 \%)$ & 2 \\
Polybacterial & $3(60.0 \%)$ & $2(40.0 \%)$ & 5 \\
Culture-negative & $3(8.6 \%)$ & $32(91.4 \%)$ & 35 \\
Total & $32(26.6 \%)$ & $92(73.4 \%)$ & 124 \\
\hline
\end{tabular}


Table 5 Comparison of outcomes in gram-positive and gram-negative peritonitis

\begin{tabular}{llll}
\hline Outcome & $\begin{array}{l}\text { Gram-positive } \\
(n=42)\end{array}$ & $\begin{array}{l}\text { Gram-negative } \\
(n=36)\end{array}$ & $p$ \\
\hline Catheter loss & $7(16.7 \%)$ & $14(38.9 \%)$ & $0.027^{*}$ \\
Shift to hemodialysis & $7(16.7 \%)$ & $10(27.8 \%)$ & 0.236 \\
\hline$* 0<0.05$ & & &
\end{tabular}

environment for PD including irregular clean water supply, and delay in seeking treatment or medical advice. This is consistent with our national registry report where the higher income group has lower risk of peritonitis [17]. Other factors such as bowel flora migration and chronic malnutrition seem less likely as most patients had normal serum albumin and potassium during initial presentation of PD peritonitis (mean of 32.19 \pm $6.45 \mathrm{mmol} / \mathrm{L}$ and $3.45 \pm 0.75 \mathrm{mmol} / \mathrm{L}$, respectively).

The current study has allowed us to evaluate the evolution of microbiology in CAPD peritonitis in our population. Over the 3-year study period, there was an increasing incidence in CAPD peritonitis in contrary to other centers which showed significant decreasing trend in incidence over years $[12,13]$. This is worrying and may be contributed by the reasons stated below but needs further analysis so that the rate of peritonitis can be reduced. We have increased intake of CAPD patients over the 3-year study period. There were 17 new patients in 2013, 28 new patients in 2014, and 42 new patients in 2015. Many of our patients are from suburban areas where the home environment may not be ideal for CAPD and a clean water supply may not be available all the time. They may also present late or do not seek advice promptly from our CAPD staff when needed. Some patients have to resort to rain water or mountain water supply at times, and some patients reported dirty water from the pipe prior to onset of peritonitis. To overcome this problem, we have started installing water filters for some of the patients. In 2013-2015, we installed water filters for three patients per year and this has increased to seven patients in 2016. Our CAPD nurses also tried to do more home visits to see how to improve patients' home environment for CAPD. This may be quite difficult and time-consuming as some of our patients live very far away in the rural areas. In 2013, 10 home visits were done and this has increased

Table 6 Association of PD catheter removal and exit-site cream among 14 episodes of Pseudomonas peritonitis

\begin{tabular}{llll}
\hline Exit-site & \multicolumn{2}{l}{ PD catheter removal } & Total \\
\cline { 2 - 3 } & Yes & No & \\
\hline Mupirocin & 3 & 5 & 8 \\
Gentamycin & 4 & 2 & 6 \\
& 7 & 7 & 14 \\
\hline
\end{tabular}

to 18 in 2014 and 29 in 2015 . All patients or their assistants were trained for 10 days to 2 weeks prior to the start of CAPD. Some of them, however, did not follow instructions strictly after discharge, and some assistants subsequently passed the "job" to other family members when they were busy. We noted quite a number of cases of peritonitis due to CAPD being performed by nontrained personnel. We have re-emphasized this during training and have done reassessment or re-training of the patients or their assistants during hospital admissions, clinic visits, or home visits. Similar problems with different magnitude have been reported in previous studies from developing countries involving CAPD in rural area [18, 19].

The increase in overall peritonitis rate in the current study was mainly due to the increase in gram-negative peritonitis while the episodes of gram-positive peritonitis remained the same. At the end of the 3-year period, gram-positive and gram-negative organisms accounted for almost equal proportions of peritonitis at our center. This trend is also noted in our national registry [17] and similar to those reported by Prasad et al. [12] and other studies [20, 21]. Most of the previous series, however, reported that gram-positive bacteria accounted for approximately two thirds of the peritonitis episodes with gram-negative bacteria accounting for one third or less of peritonitis episodes $[4,9,11,13]$.

In gram-positive single-organism peritonitis, CoNS was the most common organism isolated in many previous reports $[3,9,12,13]$. However, in our study, Streptococcus sp. was the most common among the gram-positive organisms, a finding which is quite similar to those from Spanish [8], Japanese [11], and Taiwanese [22] cohorts.

Pseudomonas sp. was the most commonly isolated organism in gram-negative peritonitis episodes in our study. This is in contrast with our national registry data and other series where E. coli was the most common pathogen in gram-negative peritonitis $[4,9,11,12,17]$. Such findings could be related to the water source among our CAPD patients. More than half of our patients lived in suburban areas where a clean water supply may not be universal and consistent, and some may be using untreated water from natural resources when the need arises.

Daily application of mupirocin or gentamicin cream to the exit site was routine practice in our patients. Overall, about one third of our CAPD patients used mupirocin and two thirds used gentamicin cream. As we did not collect data for those patients without peritonitis, we are unable to analyze whether there was any difference in the incidence of peritonitis between mupirocin or gentamicin cream users. It is interesting to note that among those patients with peritonitis, mupirocin use or gentamicin use was 
associated with almost equal proportion of grampositive and gram-negative peritonitis.

When we explore further into these 14 episodes of Pseudomonas peritonitis, current data suggests that gentamicin cream may be associated with lower incidence of Pseudomonas infection as the majority (two thirds) of our CAPD patients used gentamicin cream. However, the higher rate of catheter removal (66.4\%) among the gentamicin users vs $37.5 \%$ of mupirocin users in this subpopulation may suggest resistance of Pseudomonas to gentamicin. As the number of patients with Pseudomonas peritonitis in current study was small, this remains an interesting observation and may be an area of future research. The superiority of either exit-site mupirocin or gentamicin in preventing PD peritonitis remains the subject of further investigation even though there are more evidence showing exit-site gentamicin to be associated with lower overall peritonitis rate [23, 24].

Our culture-negative peritonitis rate was $25.8 \%$ which was comparable with the national average of $25.6 \%$ [17]. This is however high by the International Society for Peritoneal Dialysis (ISPD) standard [10]. Isolation of microorganism depends upon various factors, namely culture technique, previous antibiotic exposure, concentration of dialysate effluent, and lag times between sample collection and culturing [25, 26]. In our center. CAPD staff took PD fluid for culture for all cases with suspected peritonitis immediately before antibiotic administration. Delay in culturing might have contributed to culture-negative peritonitis as there was a small portion of patients with peritonitis who presented to the hospital after office hour. PD fluid for culture was taken, but the PD fluid was kept in the refrigerator and was sent to the laboratory the following morning. The high incidence of culture-negative peritonitis in this center could also be explained by previous antibiotic exposure. As over half of our patients came from suburban areas, they presented to the nearest district hospitals first when they were unwell and often intravenous antibiotics were started before they were transferred to our center. Hence, PD fluid culture was done after the start of antibiotics in these cases. Similar observations were reported by Kim et al. [13] and Szeto et al. [27], where the incidence of culture-negative peritonitis was high in the respective centers. PD fluid culture method is another aspect that we need to stress here as during the period of study conducted, the culture method in our hospital has not been standardized. PD fluid culture was carried out by either one of the three methods, namely direct inoculation of PD fluid into a blood culture bottle (BACTEC), direct culturing of $\mathrm{PD}$ fluid on an agar plate, or culturing the sediment after centrifuging PD fluid on an agar plate. The last method is the ISPDrecommended method [10], and we will encourage this method in order to decrease culture-negative peritonitis rate in the future.

The catheter loss rate in this study was $26.6 \%$, a rate which was high compared to previous series in which the catheter loss rate ranged between 9.8 and 20.4\% [9, 11-13, 28]. We believe that the higher rate of catheter loss in our study was mainly due to the high proportion of gram-negative peritonitis (29\% of total peritonitis episodes) especially Pseudomonas peritonitis. From our renal registry, Pseudomonas peritonitis was associated with the highest catheter loss rate excluding fungal and TB peritonitis [17]. Other contributing factors may include late presentation and treatment. Our study also confirmed that catheter loss was significantly higher in gram-negative peritonitis, as compared to gram-positive peritonitis ( 38.9 vs $16.7 \%, p=0.027$ ). This has been reported in many previous series, where gram-negative peritonitis was associated with a higher rate of antimicrobial resistance, catheter loss, shift of PD to hemodialysis, and mortality [12, 29, 30].

Some limitations of the study must be stressed. The most obvious limitation of the current study is that it is retrospective in design, and therefore, it has all the flaws and problems associated with such studies. This study was carried out on CAPD patients with peritonitis only. Therefore, the risk factors associated with developing peritonitis cannot be determined as data of CAPD patients without peritonitis were not collected. Due to various degrees of incomplete data collection, analysis on antimicrobial treatment and response was not possible, an aspect which is important in developing future guidelines in management of peritonitis. Lastly, this is a single-center study and the outcomes described here are unique to this study population.

\section{Conclusions}

In summary, this study provides timely data on emerging trends of epidemiology and microbiology of CAPD peritonitis in our population. Despite some similarities, there are also significant differences in the microbiological profiles and outcomes of our CAPD peritonitis from those of other centers. Increasing trend of peritonitis, high rates of culture negativity and PD catheter removal, and the role of exit-site cream in developing antimicrobial resistance are areas that need further evaluation and improvement in the future. Studies on risk factors of CAPD peritonitis, detailed microbiology, and antimicrobial treatment and response are warranted to further improve the outcome of CAPD patients. 


\section{Abbreviations}

CAPD: Continuous ambulatory peritoneal dialysis; CoNS: Coagulase-negative Staphylococcus; ESRD: End-stage renal disease; KPN: Klebsiella pneumoniae; PAE: Pseudomonas aeruginosa; PD: Peritoneal dialysis; SD: Standard deviation

\section{Acknowledgements}

The authors would like to thank Dr. Goh Kiang Hua, MBBS, FRCS, for the critical review and editing support of this manuscript.

\section{Funding}

The authors declare no financial disclosure.

\section{Availability of data and materials}

The datasets used in the current study are available from the corresponding author on reasonable request.

\section{Authors' contributions}

VEP was responsible for the data collection and drafted the entire manuscript. KHL and KFC participated in the design of the study and were involved in data the collection. LLSN and LWSH interpreted the results and contributed to the manuscript writing. HHC was involved in developing the study protocol and study implementation. CKC performed the data analysis and contributed to the manuscript writing. CHHT overviewed the research All authors read and approved the final manuscript.

\section{Competing interests}

The authors declare that they have no competing interests.

\section{Consent for publication}

Not applicable

\section{Ethics approval and consent to participate}

Approval for the study was obtained from the Sarawak Clinical Research Center, who waived the need for informed consent, given the purely retrospective nature of the study.

\section{Publisher's Note}

Springer Nature remains neutral with regard to jurisdictional claims in published maps and institutional affiliations.

\section{Author details}

'Department of Medicine, Sarawak General Hospital, Jalan Hospital, 93586 Kuching, Sarawak, Malaysia. ${ }^{2}$ Department of Anaesthesiology, Kuching Specialist Hospital, Kuching, Sarawak, Malaysia. ${ }^{3}$ Department of Medicine, University Malaysia Sarawak, Kota Samarahan, Sarawak, Malaysia.

\section{Received: 22 February 2017 Accepted: 16 May 2017}

\section{Published online: 16 August 2017}

\section{References}

1. Bargman JM. Advances in peritoneal dialysis: a review. Semin Dial. 2012:25:545-9.

2. USRDS: the United States Real Data System. Am J Kidney Dis. 2003; 42 Suppl 5:1-230

3. Szeto CC, Wong TY, Chow KM, Leung CB, Law MC, Wang AY. Impact of dialysis adequacy on the mortality and morbidity of anuric Chinese patients receiving continuous ambulatory peritoneal dialysis. J Am Soc Nephrol. 2001;12:355-60

4. Han SH, Lee SC, Ahn SV, Lee JE, Choi HY, Kim BS, et al. Improving outcome of CAPD: twenty-five years' experience in a single Korean center. Perit Dial Int. 2007;27:432-40.

5. Perez Font M, Rodriguez-Carmona A, Garcia-Naveiro R, Rosales M, Villaverde $P$, Valdes F. Peritonitis-related mortality in patients undergoing chronic peritoneal dialysis. Perit Dial Int. 2005:25:274-84.

6. Boudville N, Kemp A, Clayton P, Lim W, Badve SV, Hawley CM, et al. Recent peritonitis associates with mortality among patients treated with peritoneal dialysis. J Am Soc Nephrol. 2012;23:1398-405.

7. van Esch S, Krediet RT, Struijk DG. 32 years' experience of peritoneal dialysisrelated peritonitis in a university hospital. Perit Dial Int. 2014;34:162-70.
8. Lartundo JAQ, Palomar R, Dominguez-Diez A, Salas C, Ruiz-Criado J, Rodrigo $E$, et al. Microbiological profile of peritoneal dialysis peritonitis and predictors of hospitalization. Adv Perit Dial. 2011;27:38-42.

9. Ghali JR, Bannister KM, Brown FG, Rosman JB, Wiggins KJ, Johnson DW, et al. Microbiology and outcomes of peritonitis in Australian peritoneal dialysis patients. Perit Dial Int. 2011;31:651-62.

10. Li PK, Szeto CC, Piraino B, Arteaga J, Fan S, Figueiredo AE, et al. ISPD peritonitis recommendations: 2016 update on prevention and treatment. Perit Dial Int. 2016;36:481-508.

11. Higuchi C, Ito M, Masakane I, Sakura H. Peritonitis in peritoneal dialysis patients in Japan: a 2013 retrospective questionnaire survey of Japanese Society for Peritoneal Dialysis member institutions. Renal Replacement Therapy. 2016;2(2):1-8.

12. Prasad KN, Singh K, Rizwan A, Mishra P, Tiwari D, Prasad N, et al. Microbiology and outcomes of peritonitis in northern India. Perit Dial Int. 2014;34:188-94

13. Kim DK, Yoo TH, Ryu DR, Xu ZG, Kim HJ, Choi KH, et al. Changes in causative organisms and their antimicrobial susceptibilities in CAPD peritonitis: a single center's experience over one decade. Perit Dial Int. 2004;24:424-32.

14. Kanjanabuch T, Chancharoenthana W, Katavetin P, Sritippayawan S, Praditpornsilpa K, Ariyapitipan S, et al. The incidence of peritoneal dialysis-related infection in Thailand: a nationwide survey. J Med Assoc Thai. 2011:94:7-12.

15. Lee GS, Woo KT. Infection in continuous ambulatory peritoneal dialysis (CAPD): aetiology, complications and risk factors. Ann Acad Med Singapore. 1992:21:354-60.

16. Suhardjono. The development of a continuous ambulatory peritoneal dialysis program in Indonesia. Perit Dial Int. 2008;28 Suppl 3:59-62.

17. Goh BL, Ong LM, editors. Twenty second report of the Malaysian dialysis and transplant 2014, Kuala Lumpur. 2015.

18. Hyodo T, Hirawa N, Hayashi M, Than KMM, Tuyen DG, Pattanasittangkur K, et al. Present status of renal replacement therapy at 2015 in Asian countries (Myanmar, Vietnam, Thailand, China and Japan). Ren Replace Ther. 2017:3(11):1-14

19. Nayak KS, Sinoj KA, Subhramanyam SV, Mary B, Rao NW. Our experience of home visits in city and rural areas. Perit Dial Int. 2007;27 Suppl 2:27-31.

20. Zelenitsky S, Barns L, Findlay I, Alfa M, Ariano R, Fine A, Harding G. Analysis of microbiological trends in peritoneal dialysis-related peritonitis from 1991 to 1998. Am J Kidney Dis. 2000;36:1009-13.

21. Verger C, Ryckelynck JP, Duman M, Veniez G, Lobbedez T, Boulanger E, et al French peritoneal dialysis registry (RDPLF): outline and main results. Kidney Int Suppl. 2006;103:S12-20

22. Hsieh YP, Chang CC, Wang SC, Wen YK, Chiu PF, Yang Y. Predictor for and impact of high peritonitis rate in Taiwanese continuous ambulatory peritoneal dialysis patients. Int Urol Nephrol. 2015:47:183-9.

23. Bernardini J, Bender F, Florio T, Sloand J, Palmmontalbano L, Fried L, et al. Randomized, double-blind trial of antibiotic exit site cream for prevention of exit site infection in peritoneal dialysis patients. J Am Soc Nephrol. 2005;16:539-45.

24. Burkhalter F, Clemenger M, Haddoub SS, McGrory J, Hisole N, Brown E. Pseudomonas exit-site infection: treatment outcomes with topical gentamicin in addition to systemic antibiotics. Clin Kidney J. 2015:8(6):781-4.

25. Kocyigit I, Unal A, Karademir D, Bahcebasi S, Sipahioglu MH, Tokgoz B, et al. Improvement in culture-negative peritoneal dialysis-related peritonitis: a single center's experience. Perit Dial Int. 2012;32:476-8.

26. Sewell DL, Golper TA, Hulman PB, Thomas CM, West LM, Kubey WY, et al. Comparison of large volume culture to other methods for isolation of microorganisms from dialysate. Perit Dial Int. 1990;10:49-52

27. Szeto CC, Wong TYH, Chow KM, Leung CB, Li PKT. The clinical course of culture-negative peritonitis complicating peritoneal dialysis. Am J Kidney Dis. 2003:42:567-74

28. Lee S, Kim H, Kim KH, Hann HJ, Ahn HS, Kim SJ, Kang DH, Choi KB, Ryu DR Technique failure in Korean incident peritoneal dialysis patients: a national population-based study. Kidney Res Clin Pract. 2016;35:245-51.

29. Siva B, Hawley CM, McDonald SP, Brown FG, Rosman JB, Wiggins KJ, et al. Pseudomonas peritonitis in Australia: predictors, treatment, and outcomes in 191 cases. Clin J Am Soc Nephrol. 2009:4(5):957-64.

30. Jarvis EM, Hawley CM, McDonald SP, Brown FG, Rosman JB, Wiggins KJ, et al. Predictors, treatment, and outcomes of non-Pseudomonas Gram-negative peritonitis. Kidney Int. 2010;78(4):408-14. 